\title{
TREATMENT OF ASTHMA BY EXCISION OF THE CAROTID BODY
}

\author{
By J. M. LegGate, F.R.C.S. \\ 'Surgeon, Moukden Mission Hospital, Manchuria
}

In 1943 Kusinoki $^{1}$ reported five cases of asthma treated by excision of the carotid body. He found that though the attacks did not entirely disappear, they were considerably diminished both in severity and frequency. The patients themselves were very satisfied with the result. The continuous dyspnoea caused by emphysema and bronchitis was not affected, nor were the skin tests of sensitivity to allergic substances. Later the operation was performed for other conditions believed to be due to sympathetic-parasympathetic imbalance, but the results are not accessible.

In $1947 \mathrm{~S}$. C. Chen ${ }^{2}$ reported a case of a Chinese woman, aged 36 , who had suffered from asthma for I I years. After excision of the carotid body on one side she was free from symptoms for five months, when mild attacks started after a pregnancy. Later she returned for the second operation, after which she was free from asthmatic attacks for four or five months, when she again became pregnant and attacks recurred. After this, with the onset of generalized oedema and dyspnoea, she became worse and died of heart failure 1 I months after the second operation.

The following cases are reported briefly in the hope that others with greater opportunities for the investigation and follow-up of cases may attempt a larger series. In these reports emphasis has been laid on the symptomatology. All the patients were Chinese. In some cases subjective relief has been marked, but objective evidence from spirometric ${ }^{3}$ and other observations is lacking and will be necessary before the value of the operation can be truly assessed.

\section{Case Reports}

Case I. A woman, aged 53, was admitted on 5.9.47 with a history of bronchial asthma for 17 years. She had been treated as an in-patient II years before, and since then as an out-patient. For the first seven years the attacks occurred only in the winter, then in both summer and winter. For the previous six months or more she had been having an attack every afternoon, lasting till about midnight. Adrenalin and ephedrine had largely lost their effect. On examination-asthma, bronchitis. emphysema.
8.9.47. Excision of right carotid body.

9.9.47. Slept well. Much relief immediately after operation; slight giddiness present; complains of sore throat; wheezing sounds diminished.

19.9.47. Excision of left carotid body. Patient said she felt the left side of her chest become more comfortable.

30.9.47. Discharged from hospital.

8.r.48. Seen at out-patient department. Much improved. No attack of asthma since the first operation, but the cough is still present. Some wheezing sounds heard on auscultation. Patient very happy, but regrets all the money spent on medicines in the past years !

I0.9.48. No attack of dyspnoea since the operation one year ago. The cough has also improved; she lies down to sleep which she could not do for about 13 years before operation; body weight increasing; can walk and work without discomfort.

1.2.49. The above improvement has been maintained. Patient considers herself cured.

Result. No recurrence of asthma for 17 months.

Case 2. Male, aged 32, admitted to hospital, 13.9.47, with a history of cough, expectoration of frothy sputum and attacks of dyspnoea for nine years beginning in the autumn of each year. An attack usually starts in the night with a feeling of stuffiness in the nose followed by coryza, stridor and severe dyspnoea, which makes him think he is going to die. After he is able to cough up the sputum the attack subsides. There are usually two attacks each night, each lasting $\mathrm{I}_{5}$ to 30 minutes. They are relieved by adrenalin which he always has to have at hand, but it is losing its effect. Between the attacks, which last about four days, he is quite well. Some time ago he had a long series of skin tests, but no specific protein was found.

16.9.47. Excision of right carotid body. Before he was off the table he volunteered the statement that the right side of his chest felt less 'tight.' Post-operative vomiting.

17.9.47. Dizziness on sitting up. Nausea and vomiting have passed off.

23.9.47. Excision of left carotid body. On picking up the sinus nerve (it is not certain whether 
it had been adequately infiltrated by the procaine) the patient felt faint and wanted to vomit; respiration and the circulation were depressed. The symptoms passed off in 5 to ro minutes. Nausea for two hours after the operation.

25.9.47. Patient discharged as he had business to attend to.

Post-operative history. He had three recurrences within the first eight days of leaving hospital, but these attacks were mild and responded at once to adrenalin, whereas previously two injections sometimes failed to relieve the attack. Three months later (December) he had another attack, and again in the following autumn (September). The next recurrence occurred in December, 1948, and lasted five days. It was relieved, he thought, by penicillin injections which his doctor gave him. Result. Improvement.

Case 3. Female, aged 47, admitted to hospital 26.8.47, with a history of 14 years' cough, expectoration and attacks of asthma. The attacks formerly came only in the spring and summer, but now throughout the year, and they were increasing in severity and frequency. The chest tightens up, she sweats profusely, cannot get her breath and feels she is going to die. Attacks are brought on by digestive troubles, emotional disturbance or overwork. On examination-emphysema, bronchitis, orthopnoea.

22.9.47 and 1.10.47. Excision of right and left left carotid bodies. Post-operative sore throat and giddiness.

13.10.47. The cough and expectoration have not been improved, and attacks of dyspnoea still occur at night, but milder, to the extent that she is now comfortable lying down. She herself is very satisfied.

7.1.49. Patient writes that in the last 12 months she has had about ten attacks of asthma. They have been milder than before operation. The continuous dyspnoea between the attacks and the cough are the same as before.

Result. Probably some improvement.

Case 4. Female, aged 21 , admitted 23.9.47. 'Two years' history of typical asthma; attacks brought on, she thought, by petrol vapour and salty foods.

26.9.47 and 3.10.47. Excision of carotid bodies.

4.10.47. Feels tightness of the chest and is dyspnoeic.

5.10.47 and 6.10.47. Severe attacks; orthopnoea.

8.i I.47. Miscarriage at seven months. Asthma as bad as ever.

Result. No improvement.
Case 5. Male, aged 54, admitted 21.11.47. complaining of cough, frothy expectoration and paroxysmal dyspnoea for between ro and 20 years, worse in the winter. Now attacks come on every night accompanied by profuse sweating and much sputum. Adopts the knee-elbow position for relief. Some benefit obtained with ephedrine. On examination-bronchiectasis and emphysema.

25.11.47 and 12.12.47. Excision of carotid bodies. Post-operative. Had a sensation of phlegm rattling in his throat. This passed off in two or three days.

22.12.47. Discharged. Cough and sputum have continued as before, but he has had no attacks of asthma since the operation. $\mathrm{He}$ feels his breathing freer.

Result. Improved while in hospital but no follow-up.

Case 6. Soldier, aged 25, admitted 19.7.48. Attacks of asthma in the spring and summer for six years. Now they occur on an average every week and last one to three days. He does not know what brings on an attack. Adrenalin is of benefit.

20.7.48 and 27.7.48. Excision of carotid bodies,

21.7.48. A little difficulty in swallowinge passed off in the evening.

25.7.48. Typical attack of asthma started a和 midnight with sweating and cyanosis. The sweating was worse than usual.

30.7.48. Discharged. Later he wrote to say that after leaving hospital the cough became worse and the amount of sputum increased. Attacks of asthma occurred on August 7, 16, 22, 27 and September 7 .

Result. No improvement.

Case 7. Female, aged 34, very poor and undernourished. Cough and dyspnoea for seven years, at first only in the autumn and winter but now all the year round. Has been off work for two years. Every day from 7 to 10 a.m. and again from 4 to 12 p.m. she has severe expiratory dyspnoea: adopts the knee-elbow position with her forehead resting on her fists. She cannot move until the attack is over. If she walked as far as her outside lavatory it brought on an attack, and it was some considerable time before she could speak for $N$ breathlessness. On examination-marked em- N physema and bronchitis. Orthopnoea.

I.10.48. Excision of right carotid body. The first two nights after the operation the patient $\frac{}{\Phi}$ lay down and slept comfortably; complained of $\stackrel{\mathcal{Q}}{?}$ sore throat. The third night the cough was very ${ }^{\circ}$ troublesome and it was accompanied by wheezing, but the cough was not followed by an attack of dyspnoea as it was before operation. 
6.10.48. Excision of left carotid body. During the operation on the right side the B.P. had gone up from $114 / 84$ to $160 / 100$ on excision of the carotid body, returning to $\mathrm{r} 34 / 84$ in 20 minutes. It should be mentioned that the normal B.P. of the Chinese is lower than that of Westerners, but data is lacking as to the normal. During the second operation there was a rise from $\mathrm{I}_{5} / 80$ to $140 / 96$, but thereafter it fell steadily until 26 hours later it was $84 / 50$ and there was nausea, vomiting and left temporal headache; 48 hours after the operation it had returned to 105/70, and on discharge from hospital on $14 \cdot 10.48$, to the preoperative figure. While in hospital she was able to lie ' on her side or on her back' for the first time for two years. Cough, cyanosis, expectoration and some dyspnoea of effort were still present, but there were no bad attacks of bronchial spasm.

22.10.48. Walks six miles to hospital and back. She is still cyanosed and 'wheezy' with moist rales heard over both bases, but she says her breathlessness is now no worse at the end of her long walk than when she sets out. She still has occasional pain in the left side of her head.

Result. Much improved.

Case 8. Female, aged 45, admitted 1.10.48 complaining of recurrent attacks of cough and expiratory dyspnoea for 20 years, chiefly in the autumn and winter. For the last two months she could only sleep kneeling in bed with her forehead resting on a high pillow. Some relief is obtained with adrenalin. On examination-wheezing, cyanosis, much mucoid sputum, orthopnoea, emphysema.

4.10.48. Excision of left carotid body.

5.ro.48. Patient slept quite well lying flat for the first time for two months.

II.10.48. Excision of right carotid body. The small hook passed round the intercarotid bundle may have included the ascending pharyngeal artery or the body itself may have had an artery of similar size passing into it, for as it was excised there was an unusually brisk spurt of haemorrhage. This was controlled by the finger until slings had been passed under the common and external carotid arteries. This enabled the haemorrhage to be controlled while the bleeding point was picked up with ' mosquito' forceps. 'The stump was too short to ligature and the 'mosquito' forceps were left in situ for 48 hours and removed without further trouble. The microscope confirmed the excision as usual.

20.1.49. There has been no recurrence of the asthma. She lies down comfortably at night, is eating very well and obviously putting on weight. The cough has improved but she still requires her Tinct. Camph. Co. On auscultation-marked emphysematous breathing but no wheezing. She is very happy with the result.

Result. Much improved.

Case 9. Female, aged 39, though looks older. Ten years' history of cough, palpitations and attacks of dyspnoea, increasing in severity, always worse in winter. Sometimes during the last four years she has noticed oedema of the ankles, hand and face. On examination-orthopnoea, cyanosis, bronchitis and emphysema; distension of the veins of the neck; B.P. 110/70.

i.I1.48. Section of the right sinus nerve immediately above the carotid body. Atropine, gr. $1 / 100$, and morphia, gr. $\frac{1}{6}$, were given one hour before operation, and the morphia, gr. $\frac{1}{6}$, repeated at the commencement of the operation. The table was tilted slightly head-up, but this failed to relieve the congestion of the veins of the neck due to the heart condition. The sinus nerve bundle, after infiltration with procaine, was divided between fine cotton ligatures, the lower one passing round the carotid body and the upper one above it. The patient was very comfortable after the operation and said she felt her breathing 'stronger.'

On her return to the ward she slept, but latero in the afternoon she was found to be semi comatose. The respiratory and vasomotor centreso were profoundly depressed. At 4 p.m. (five hours after the operation) the B.P. was $82 / 30$. By 5 p.m. it had fallen to 48 systolic. The diastolic was difficult to ascertain as the sounds could be heard down to zero. The respiration fell to five to eight per minute, with short inspirations and long pauses in expiration. The pulse rate remained about 8o. There were pin-point pupils and cyanosis of the lips. Movements of the jaw as in gasping were still present showing that the carotidmandibular reflex was still present owing to the intact left carotid body ${ }^{8}$. Oxygen was given and injections of nikethamide, camphor and atropine. Atropine, gr. $1 / 100$, given intravenously failed to dilate the pupils. By 6 p.m. the B.P. had recovered to $64 / 24$, pulse rate 100 . Next morning the patient had regained consciousness, and complained of nausea and vomiting (even of water), B.P. $60 / 40$, respiration 18 , pulse 100. In the afternoon the B.P. was $74 / 30$ and the patient felt comfortable. She got up and walked to the basement during an air raid, but afterwards had to be carried back. Feels pain in her throat on swallowing solid food.

3.1 1.48. If she tries to sit up she feels her head is going to burst. B.P. $80 / 20$ lying down, 65/25 sitting up in bed. Sputum much more easily expectorated. Sleeps and breathes easily lying down (she says she has not spent a whole night 
lying down for about ten years). She has taken ephedrine for seven years, now does not feel she needs it.

5.1 1.48. B.P. $86 / 55$ (lying). In the evening she had a mild recurrence of dyspnoea but she could lie down during the attack. Cough more troublesome. Wheezing sounds heard on both sides of the chest.

8.1 1.48. Got up during an air raid in the night. Signs of right heart failure; oedema of the hands and sacral region, palpitation and dyspnoea.

10.I I.48. Adrenalin required and very effective.

13.11.48. Still has pain in the throat on swallowing, which sometimes passes up to the ear. Patient is satisfied her breathing is much easier (' half better'). There has been no recurrence of the severe attacks of dyspnoea, but there is still shortness of breath and slight bedema. Some wheezing is still present, but before operation it ' used to be so loud it kept people awake.' Discharged from hospital.

It is not considered advisable to operate on the other side in this case on account of heart failure. The B.P. (93/52) has not returned to the preoperation figure.

30.I I.48. Re-admitted. After leaving hospital she felt better than she had done for many years. She had had no attack of dyspnoea for ten days and her friends were congratulating her on the result. She set out with a friend to report to hospital but it started to rain and she had to hurry back home. This brought on an attack of palpitation and precordial pain, with cough and dyspnoea. Within three days her face and body were oedematous. It would seem as if her comparative freedom from respiratory distress gave her a false idea of her strength and the overexertion resulted in heart failure. On examination - generalized odema, distension of the veins of the neck, epistaxis, liver enlarged, B.P. 90/55; her dyspnoea is not marked and she lies down comfortably in bed.

She was in hospital for six weeks and her symptoms improved under medical treatment, but it was evident that the operation, while relieving the asthmatic attacks, had caused no improvement in her general condition. She was very desirous for the left side to be operated on as she thought that the left side of her chest felt 'tighter' than the right, but it was considered that the further depression of the circulation resulting from the bilateral operation would be serious and far outweigh any benefit of the effect of the respiratory system.

Result. Asthma improved but symptoms of heart failure increased.

A tenth case has been done too recently for the result to be assessed, though the immediate effect was satisfactory.

The patient was a man, aged 4I, with six years' history of asthma associated with bronchitis, and bilateral section of the sinus nerve was performed. $\mathrm{He}$ had required injections of adrenalin every night for the seven nights preceding the first operation, when the attack ceased, but this may just have happened to coincide with the natural termination of the attack.

\section{Comment}

This series is too small for definite conclusions to be drawn, but the following are suggested:(I) Until a more satisfactory basis for the selection of cases can be arrived at, results are likely to be very variable. (2) The younger patients whose asthma is more specifically allergic derived little or no benefit from the operation. (3) Elderly patients with a long history of asthma associated with bronchitis and emphysema derived considerable benefit. The Japanese article referred to would have led us to expect the contrary. (4) The presence of heart failure seems to be a contraindication to the operation.

\section{The Operation}

Local analgesia has been used in all cases. The best premedication is still undecided, but it shouldo include atropine. Oxygen is administered throughout.

An incision is made below the angle of the jaw in the line of a skin crease, crossing obliquely the anterior border of the sternomastoid muscle. The tendency is to make the incision too low. The bifurcation is exposed, care being taken to preserve the descendens hypoglossi nerve. The sheath and inter-carotid bundle are infiltrated with procaine. It has been found convenient to divide the superior thyroid artery, in one case also the lingual, so that the bifurcation may be rotated outwards. The weight of a pair of artery forceps which has picked up the sheath on the inner side of the common carotid artery (not the stump of the superior thyroid artery) will maintain the rotation for the remainder of the dissection. The carotid body is slightly larger than a pin head $(5 \times 3 \times 2$ $\mathrm{mm}$. $)^{4}$ and will be found firmly attached to the deep aspect of the bifurcation. The inter-carotid bundle containing the sinus nerve is picked up on a blunt hook or aneurysm needle and divided. The lower end is held in 'mosquito' forceps and the carotid body freed by sharp dissection from the wall of the artery. A little bleeding occurs because the body has a rich arterial supply, but finger pressure for a minute or two is sufficient to stop it.

In the first eight cases bilateral excision was 
carried out so that the microscope could confirm the excision, but in Case 9 section of the nervous connections of the carotid body (a simpler operation than excision) was performed. Provided regeneration can be guarded against this will probably prove as effective as excision. In cases of carotid sinus syndrome (syncopal attacks due to hypersensitive sinus), section of the sinus nerve is sometimes followed by a recurrence of the symptoms $^{5}$ owing to the difficulty of completely denervating the sinus, for twigs from the internal carotid artery plexus join the sinus nerve at a high level ${ }^{6}$. The chemo-receptors in contradistinction to the presso-receptors, are concentrated in the carotid body and should therefore be more easily denervated. But the operation can never completely eliminate chemo-receptor influence, for the aortic body remains.

Post-operative pain in the throat, probably glosso-pharyngeal in origin, is commonly complained of for one or two days, and in one case pain in the temporal region-perhaps trigeminal ${ }^{6}$, but the explanation of these referred pains is not clear. In some cases there has been dizziness on sitting up, which has lasted for one or two days, due to interference with the blood pressure adjustment to posture, but such complaints have not been as marked as might have been expected. There have been no consistent changes in the pulse or respiration rate, or in breath-holding time, and even the rise of blood pressure, due to elimination of the presso-receptor reflexes, has been very variable both in degree and duration.

In Cases 2, 7 and 9 there were disturbing signs of medullary depression, which at first seemed to point to a type of carotid sinus reflex, due to stimulation of the sinus nerve, in the first by the forceps and in the other two by a ligature. However, the probable explanation is an increased sensitivity of the medullary centres to the procaine and the morphia. When the centres are under the influence of a narcotic, they become more dependent on the carotid body mechanism, so that after denervation the narcotic has an exaggerated effect. This has a bearing on the question of the choice of anaesthetic. In Case 9 two injections of morphia, gr. $\frac{1}{6}$, with an hour's interval in between, nearly proved fatal, but in this case cardiac failure was a factor of importance. It should be added that ampoules of morphia from the same batch were given to other patients without untoward effect, showing there had been no mistake in the dosage.

A somewhat similar collapse was seen in a case of carotid sinus syndrome reported by Ray and Stewart ${ }^{5}$. 'Three months after denervation of the right carotid sinus by intra-cranial section of the glosso-pharyngeal nerve, the left carotid sinus region was infiltrated with $24 \mathrm{cc}$. of I per cent. procaine. The blood pressure rose abruptly from $170 / 100$ to $236 / 120$, then shortly dropped to $75 / 50$, followed by pallor, sweating and mental confusion. After 20 minutes the symptoms passed and the B.P. rose to $240 / 120$. The neurological signs of complete anaesthetization of the carotid sinus region were still present.' This collapse was attributed to the procaine.

\section{Discussion}

With so many unknown factors the field for vain speculation is a wide one, but some attempt to explain the rationale of the operation is due.

'The carotid and aortic bodies owe their function to the presence within them of nerve-endings specialized to respond to certain changes in the chemical composition of arterial blood, such changes giving rise to reflexes which can produce physiologically important effects upon respiration and circulation.' 7 These chemo-receptors are stimulated by anoxaemia, a lowered $\mathrm{pH}, \mathrm{CO}_{2}$ excess, choline derivatives and other substances, and stimulation causes hyperpnoea. In fact, the stimulant effect of anoxaemia on respiration is almost entirely reflex from the carotid and aortic bodies. Section of the sinus and depressor nerves, in experiments on animals, changes the violent hyperpnoea produced by anoxaemia into a veryo slight respiratory stimulation. ${ }^{7}$ The carotid bodies are also concerned in certain reflexes which are brought into action during acute respiratory failure due to anoxaemia, such as the "gasp" reflex (carotid-mandibular). ${ }^{8}$ 'There is evidence also that chemo-receptor reflexes may have a farreaching influence throughout the central nervous axis,' Kaufman.'

Removal or denervation of the carotid body in cases of asthma might be expected to diminish the acute attacks of dyspnoea either centrally, by preventing certain stimuli from reaching the respiratory centre, or peripherally, by causing reflex relaxation of the bronchial spasm, via the vagus or sympathetic connections. The latter, it would seem, is the view held by Kusinoki.

However, since (I) anoxaemia is the chief stimulus of the carotid bodies, and (2) anoxaemia was probably present in those cases which benefited from the operation; it is suggested that the primary result of operation is to relieve the centre of the reflex effects of anoxaemia.

In cases such as 4 and 6 , where the allergic factors were predominant and there were few permanent changes in the lungs to cause anoxaemia, the operation produced no benefit. This seems to indicate that no essential reflex of the asthma complex is interrupted by the operation. In cases complicated by chronic bronchitis 
and emphysema the tendency to anoxaemia is greater, and so the primitive mechanism of the carotid bodies plays a larger part in the acute attacks of dyspnoea than in cases of purely allergic asthma. Cyanosis is an unreliable guide in the recognition of arterial anoxaemia. Comroe and Botelho, ${ }^{\circ}$ using a photo-electric colorimeter (oximeter) found that the majority of observers are unable to detect cyanosis until the oxygen saturation has fallen to 80 per cent., so that the threshold of sensitivity of the carotid bodies to anoxaemia? may be passed before cyanosis is noticeable.

But even if it be granted that there is some degree of anoxaemia in these cases, that the carotid bodies are stimulated thereby and that their removal would relieve the centre of what, in the circumstances, is only an embarrassing bombardment of stimuli, is there any evidence that anoxaemia can initiate the episodes of bronchial spasm?

One must presume an inherent susceptibility to broncho-spasm, a predisposition of the lungs to respond in this abnormal way to stimuli. No operation could be expected to alter this fundamental sensitivity. Even after bilateral resection of the posterior pulmonary plexus, attacks of asthma can recur. ${ }^{10}$ But what can be expected from the removal of the carotid bodies is an increased tolerance to anoxaemia. It has been found that in those cases which have been improved by operation, the symptom most relieved is orthopnoea. Patients feel they can go to sleep lying down without the fear of being wakened up by a severe attack of asthma. The cause of these night attacks is not known, but it is reasonable to suppose that in the recumbent position the diminished excursion of the diaphragm and hypostatic congestion leads to a reduction of oxygenation. Attacks brought on by exertion might also be explained by reduction in oxygen pressure. Further, 'in some cases of bronchial asthma, not only does the administration of oxygen relieve the anoxaemia ... but also it tends to relieve the broncho-spasm.' ${ }^{11}$ May this not be because anoxaemia is a precipitating cause in such cases ? not indeed that anoxaemia per se initiates the attack, but rather that the fall in oxygen tension from its previous level, so to speak, pulls the trigger.

When the anoxaemia reaches the threshold of sensitivity of the chemo-receptors, they respond strongly and the centre reacts, normally by hyperpnoea, but in sensitive cases this brings on an asthmatic attack.

Though this hypothesis of anoxaemia as a precipitating factor is largely conjecture, there can be little doubt that anoxaemia plays a part in the established asthma attack, and therefore that the operation, by eliminating one source of stimuli, might diminish the severity of an attack.

The centre is the weakest link in the reflex chain, and there comes a point when it fails to maintain the balance between the nervous and chemical stimuli. Normal respiratory reflexes (HeringBreuer) become inco-ordinated and abnormal ones may become manifest. It is suggested that the operation, by eliminating the stimuli from the carotid bodies caused by anoxaemia, diminishes the fatigue of the centre and so delays the onset of asphyxial symptoms. It stops the whipping of the tired horse and the anoxaemia, instead of stimulating respiration to the point of paroxysm, may even depress respiration by direct action on the centre.

Unfortunately, the cases operated on so far have not been investigated pre-operatively from the point of view of their response to oxygen administration, for it was not until the results were seen and co-related with what is known of carotid body function that anoxaemia came to be considered the factor of importance here suggested.

However, in addition to this effect on the centre there is a possibility that denervation of the carotid bodies leads to some reflex relaxation of bronchial spasm. The sinus nerve to which the fibres from both the carotid body and carotid sinus pass joins the glosso-pharyngeal nerve, but connections are also made with the superior sympathetic ganglion? and the vagus nerve. It appears that although the impulses from the carotid sinus (presso-receptors) are transmitted by the glosso-pharyngeal nerve, those from the carotid body (chemo-receptors) may pass by one of the other connections. ${ }^{5}$ Stimulation of both presso- and chemo-receptors leads to cardiac inhibition by vagal reflex. In some cases of carotid sinus syndrome and carotid body tumour ${ }^{12}$ there is increased vagotonia which is relieved by section of the sinus nerve or removal of the tumour. It is possible, therefore, that the abnormal vagotonia seen in asthma might be affected by section of the sinus nerve.

In favour of some such peripheral relaxation is the observation that (I) in some cases the patient volunteers the statement, before he is off the operating table, that one side of his chest feels less ' tight,' and (2) that auscultation sometimes reveals a diminution of wheezing on that side, such as has been reported to occur after procaine block of the vagus nerve. ${ }^{13}$

It has been noticed also, that in those cases which have recurred after operation, there has been an improved response to adrenalin, which suggests that the operation may possibly result in an increased sensitivity of the bronchial musculature to circulating adrenalin, but this is speculative.

\section{$\overrightarrow{0}$}

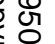


It will be evident that the operation raises more questions than it answers, but whatever the explanation it does seem to be effective in alleviating the attacks of asthma in some cases. It deserves wider investigation.

\section{Summary}

Eight cases of asthma of various types have been treated by bilateral excision of the carotid body, and one by unilateral denervation. Those associated with chronic bronchitis and emphysema have been improved rather than those of typically allergic asthma.

\section{Acknowledgment}

My thanks are due to Dr. S. C. Chen for her help with these cases and with the translation of the Chinese and Japanese articles.

\section{REFERENCES}

I. Kyushu Univ. Med. F., November, 1943 (Japanese)

2. Moukden Med. Col. F., October, 1947 (Chinese)

3. LEVY and SEABURY (1947), $\mathcal{F}$. of Allergy, 18, 244

4. LECOMPTE, P. M. (1948), Am. F. Path., 24, 305.

5. RAY, B. S., and STEWART, H. J; (r 948), ' Glossopharyngeal

Nerve in Carotid Sinus Reflex,' Surgery, 23, 4I I.

6. TCHIBUKMACHER, N. B. (1938), 'Surg. Anat. of Carotid Sinus Nerve' Surg. Gyn. Obst., 67,740 .

7. SCHMIDT, C. F., and COMROE, J. H. (1940), ' Function of Carotid Body,' Phys. Rev., 20, 1 I 5.

8. TSCHIRGI, R. D., and GERARD, R. W. (1947), 'Gasp Reflex,'Am. F. Phys., r 50, 358 .

9. COMROE, J. H., and BOTELHO, S. (1947), Am. Y. Med.

ro. RIENHOFF, W. F., and GAY, L. N. (1938), Arch. Surg. Chicago, 37, 456, quoted in Brit. Enc. Med. Prac. S., r939, p. 232.

I. CHRISTIE, R. V. (1937), Brit. Enc. Med. Pract., 4, 395.

12. COKE, M., and DUNLOP, H. A. (1932), Lancet, 2, 1050.

13. WEISS, S., and ROBB, G. P. (I933), 'Cardiac Asthma, Ұ.A.M.A., 100, 1845 .

\title{
MARROW PUNCTURE AND ITS AID IN DIAGNOSIS
}

\author{
By Angus E. Brewer, M.R.C.S. \\ Clinical Pathologist, Hampstead General Hospital, Royal. Free Hospital Group
}

A patient's blood count reflects the condition and the function of the bone marrow. An abnormal blood picture that is inadequately explained by the clinical condition is a direct indication to examine the marrow.

The first attempt at marrow biopsy was made at the beginning of this century by trephining the femoral epiphysis or the upper third of the tibia. The inconstant presence of active marrow at these sites produced indifferent results and it was not until Seyfarth described trephining of the sternum in 1923 that widespread interest was founded. Trephining is somewhat laborious and the procedure was much simplified when Arinkin ${ }^{1}$ in 1927 showed that suitable material for examination might be obtained by puncturing the sternum with a wide gauge needle.

Since then many varieties of needle consisting of a trochar and cannula have been designed. The Salah needle is now in general use and has an adjustable stop which acts as a safeguard against over-penetration and enables the operator to gauge the depth of the needle point.

The site usually chosen for puncture is the manubrium or the body of the sternum between the second and the third rib in the mid-line. Recently successful puncture of the spines of the vertebrae, the iliac crests and the ribs has been described and the marrow thus obtained in normal subjects has been identical with that from the sternum $^{2} 34$.
The patient is given a sedative and lies flat in the bed. The skin and periosteum are infiltrated with 2 per cent. novocain. The needle is pushed vertically through the skin and into the bone with a firm boring motion. The amount of pressure necessary will depend on the thickness and the condition of the bone. A give is usually felt as the marrow cavity is entered. The stylet is withdrawn and a $2 \mathrm{cc}$. sterile paraffined syringe attached and 0.2 cc. only of marrow aspirated, thus preventing excessive dilution with blood. The stylet is replaced, the needle withdrawn and the wound sealed with collodion. The patient should then be at rest in bed for some hours.

\section{The Dangers of Sternal Puncture}

Haemorrhage and haematoma may occur, particularly in blood dyscrasias, but are never serious unless the patient has been allowed up immediately. Very rarely death from shock has occurred and adequate sedation would seem to be a necessary precaution. Infection, a potential possibility, has not been noted. Puncture of the heart has only been reported where an unguarded or unsuitable needle has been used.

It has been stated that haemophilia is the only contraindication.

\section{Normal Marrow and the Limitations of the Biopsy}

Normal marrow is semifluid and consists of 